\title{
Design and Implementation of Sensor for Velocity of Detonation Measurement
}

\author{
Aruna D. Tete ${ }^{\# 1}$, Dr. A. Y.Deshmukh ${ }^{* 2,}$ Dr. R. R. Yerpude ${ }^{\# 3}$ \\ ${ }^{\# 1}$ Department of Electronics Engineering, G.H.Raisoni College of Engineering, Nagpur,India \\ ${ }^{* 2}$ Department of Electronics Engineering, G.H.Raisoni College of Engineering, Nagpur,India \\ ${ }^{* 3}$ Department of Mining Engineering, Visvesvaraya National Institute of Technology, Nagpur India
}

\begin{abstract}
Velocity of Detonation (VOD) is the most important property to consider when rating an explosive. It is an established fact that VOD gives a good indication of the strength and hence the performance of the explosive. Detonation Sensor is the most critical part of the VOD measurement system. This paper presents and discusses various practical and field implemental techniques for sensing detonation wave with detailed study of electrical ionization principle using resistance sensor for VOD measurement. In this technique effort is made to overcome the limitation of continuous wire sensor and discrete points sensor under various practical conditions. Step change in resistance is utilised to increase the system reliability, improve noise immunity as well as to reduce the system complexity.
\end{abstract}

Keywords: Discrete resistance, Explosive, Ionization, Picoscope, Velocity of Detonation

\section{Introduction}

Velocity of Detonation (VOD) is an important characteristic measure of explosive materials. When new explosives are developed, their VOD must be determined. Evaluation of new explosive composition is an important part of their development. Velocity determination is made by measuring the time required for the detonation wave to travel a measured distance longitudinally through a column of the explosive. The detonation wave travels at speeds much higher than speed of sound. Typical detonation velocities for commercial explosives range from $2500 \mathrm{~m} / \mathrm{sec}$ to $7000 \mathrm{~m} / \mathrm{sec}$. The detonation velocity is amongst the most important property of the explosive and very difficult and tricky to measure. The velocity of detonation of a particular explosive depends on factors such as 1) charge diameter 2) confinement 3) density 4) primer size and type 5) Density etc. It may be expressed as a confined value and unconfined value and is normally given in meter per second $(\mathrm{m} / \mathrm{s})$ or feet per second $(\mathrm{fps})[3]$. The confined detonation velocity measures the speed at which the detonation wave travels through a column of explosive within a borehole or other confined space. The unconfined velocity indicates this rate when the explosive is detonated in the open. The measurement of the VOD in column of explosive placed in the ground or in various cylindrical containers (cardboard, PVC, steel) mainly relies on estimating the time required for detonation wave to propagate a given distance.

\subsection{Dynamics of explosion and its significance in sensor design.}

Study of Dynamics of explosion reveals that during explosion following things take place -

- $\quad$ Light emission - infrared, visible, ultraviolet

- $\quad$ Sound

- Pressure - Shock wave

- Air ionization

- High temperature

Except sound parameter all other parameters progress in synchronism with the detonation wave. Thus any of these parameters can be used for determination of Velocity of Detonation (VOD). The sensors that detect the detonation front rely on various physical mechanisms such as the light produced at or near the detonation front, the pressure that causes a continuity or discontinuity in the impedance of a sensing cable or some form of electrical continuity due to air ionization because of explosion or disruption produced by the propagating front. Such measurements provide data which is plotted against the time period of the in-hole explosion and thus the VOD can be calculated. It has been observed that most of the manufacturers and the utilities rely on calculations based the chemical composition of the bulk explosive to arrive at the VOD value. Needless to mention such interpretation can be fairly inaccurate due to variations in raw material quality, manufacturing process followed and more importantly it is still a theoretical value only. Deriving the correct value of VOD with the proper measurement techniques will result in reduction in the consumption of explosives with optimized results. In this paper a sensor design approach towards a VOD measurement is discussed which includes continuous wire discrete resistance type VOD measurement technique. In this technique step change in resistance is preferred to increase the system reliability. This sensor is a low cost sensor, is easy to install/ setup, has reduced 
susceptibility to electrical noise. An ideal sensor should have large change in resistance for the given blast length. Sensor should be based on change in resistance either continuous or in steps. Step change in resistance is preferred as it reduces the system complexity as well as increase reliability. Use of only a particular grade of cable should not be a prerequisite i.e. system should be able to accommodate at least a certain range of cable/wire types. Sensor system should be flexible enough to be suitable for explosive length from $10 \mathrm{~cm}$ to $10 \mathrm{mtrs}$ moreover it should be easy to setup and connect. Sensor and it's acquisition unit should have self diagnostic feature to detect sensor fault. As noise is generated during explosion, so sensor and it's acquisition system should minimize noise interference. Sensor should not be affected by presence of moisture or other conducting media.

\subsection{Factors Affecting Sensor Design}

During explosion the detonation wave creates and causes high temperature, very high pressure, ionization of air and emission of bright light. This physical phenomenon progresses along the explosive column at same velocity as that of the detonation wave. However there could be variation in the amplitude or intensity of these physical parameters. Sensors can be based on any one of these parameters like temperature, pressure, air ionization and light. There could be a time lag in sensing and measurement process using these sensors and may vary from sensor to sensor. Sensor based on light is the fastest one with no time lag. Air ionization in the next best option as far as time lag is concerned with pressure and temperature based ones being with increased time lag. Since light sensors are difficult to implement practically and are costlier one, air ionization principle for sensor design has been chosen. This sensor makes use of electrical conductivity provided by ionized air in the detonation wave which facilitates current flow in the circuit which in normal circumstances (no detonation) acts as an insulator.

\subsection{Factors Affecting Air Ionization Based Sensor Design}

Air ionization level provided by detonation wave of explosive vary greatly. It primarily depends on

- Chemical composition of explosive.

- Diameter of explosive column.

- Density of explosive column

- Weight of explosive column.

- Moisture level or water content in explosive column

- Ambient temperature and humidity.

- Physical layout of sensor. Value, number and type of resistors used in the sensor.

- Voltage and current level permitted as per safety standards

- Physical form, shape, size, volume and weight of the sensor permissible as per safety standards and practically possible.

The proposed sensor design consists of string of resistors connected in series which gets shorted with return path progressively by the detonation wave. Thus the resistance of the sensor circuit reduces progressively causing a decrease in voltage across the sensor. The rate of decrease of this voltage corresponds to velocity of Detonation (VOD).

\subsection{Factors considered for Design of Proposed Sensor.}

Factors to be considered for design of discrete string of resistors type air ionization sensor are

- Length of explosive column under test.

- Positioning of sensor along the explosive column. The sensor has to be placed at least at one and half times the diameter of the explosive column also it has to be parallel to the explosive column.

- Conductivity levels provided by ionized air for all types of explosives. The sensors must be suitable for operation for this range of conductivity levels.

- Sensor should be moisture and water proof.

- Any means provided for moisture protection should be just adequate enough. Less protection will make sensor prone to change in current levels and hence measurements, while more protection may prevent the paths to get shorted effectively.

- Distance between path of resistor string and return path.

- Distance between the resistors.

- Current levels in the circuits.

- Value and type of resistors used in sensor design 


\section{Vod Measuring Technique}

Existing practices for measurement of VOD adapted worldwide can be broadly classified into methods listed below.

\subsection{Dautriche method}

This is one of the oldest methods, very easy to implement and reasonably accurate and used very widely all over the world. In this method Detonating Fuse (DF) of length about 1 meter whose mid point is marked is placed and bound to an aluminum plate with this centre point marked on the plate at one end[2]. The ends of this DF are inserted in the explosive cartridge at a distance of $10 \mathrm{~cm}$ from each other. The detonation wave in the explosive cartridge initiates the two DF ends sequentially. The shock wave thus induced in DF collide at some point on the aluminum plate. The distance of collision mark from mid point is measured. This distance is directly proportional to Velocity of Detonation. The formula is VOD $=\mathrm{V} \times(\mathrm{m} / 2 \mathrm{a})$

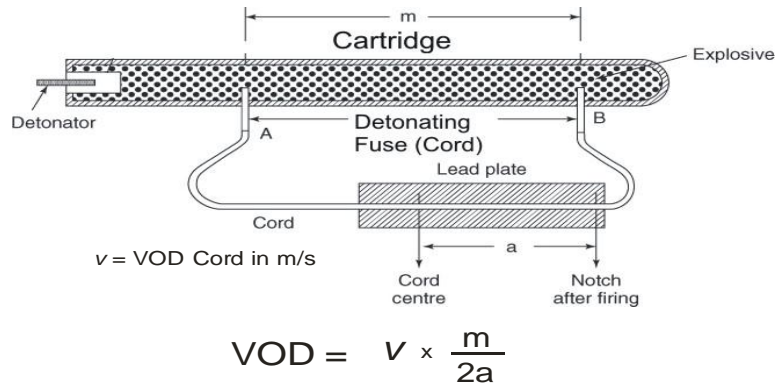

Fig. 1 D'autriche method Schematic

This method of VOD measurement is suitable for unconfined measurements only. See Fig. 2 below.

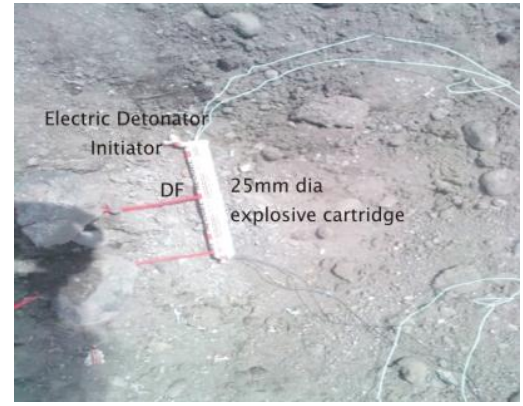

Fig. 2 D'autriche method in field Fig. 2 D'autriche method in field

\subsection{Discrete point VOD systems}

These are basically supported by electronics start and stop timer. The one end of sensor cables are inserted into the explosive column at predetermined fixed distance and other end to the VOD recorder where the start and stop signals are recorded. When detonation reaches the first sensor timer clock is started and on reaching the next sensor this clock is stopped. The timer interval $(t)$ is recorded. Since the distance (d) between sensors is known, ' $\mathrm{d} / \mathrm{t}$ ' directly gives the VOD.

\subsection{Resistance wire continuous VOD system.}

Continuous resistance wire VOD measurement system basically follow the Ohm's Law. In this method pressure caused by explosion provides electric short continuously along the sensor path, causing a reduction in sensor length and hence a continuous voltage drop under constant current excitation. The instantaneous measurement and recording of this voltage drop with respect to time gives the VOD at any instant. Thus a continuous VOD record can be obtained.

\section{$2.4 \quad$ Time Domain Reflectometer}

In this system narrow electric pulse is sent through the cable sensor and the return or reflected pulse from the end of cable is detected. This method does not require the sensing cable to be shorted in order to acquire data. This is one of the safest method to use with any commercial or military explosive. This method is suitable for measuring the VOD in multiple holes within a blast provided due care is taken with respect to both the initiation sequence and the possibility of cut-offs that may disrupt the coaxial cable. Some experimentation has been carried out with VOD sensors which was constructed by threading a high resistance $\mathrm{Ni}-\mathrm{Cr}$ wire 
through a $1.6 \mathrm{~mm}$ diameter, $90 \mathrm{~cm}$ long brass tube and crimping both at one end to establish electrical connection, with the brass tube acting as a return lead. The brass sensors generated VOD traces that were discontinuous and electrically noisy due to intermittent opening/closing of the contact making the record difficult to interpret. In order to reduce the bias in the interpretation of VOD traces, brass tubes were replaced by smaller diameter $(0.8 \mathrm{~mm})$ much softer aluminum tubes. Construction of aluminum sensor was time consuming due to easiness by which they kinked when slight pressure was applied during assembly preventing threading of the Ni-Cr wire within the tube. To avoid damaging the aluminum tube, a regular brass tube was taped alongside to provide structural rigidity.

\title{
2.5 Sliffer continuous VOD system
}

This system consist of a shorted length of coaxial cable as a sensor in the explosive column which is connected to the oscillator circuit. This small device show the frequency which is controlled by length of sensor in the explosive column. As the wire length decreases frequency of oscillation increases. By monitoring this frequency as a function of time, the rate of cable length change can be determined, leading directly the measurement of VOD. This system has been limited to laboratory work. The restriction with the SLIFER system is that recording cable length with the oscillator is $66 \mathrm{~m}$ per channel, moreover each sensor must have oscillator connected in a line which should be placed close to the hole or shot area.

\subsection{Method based on fiber-optic}

In this method optical fiber is used which is capable of detecting and transmitting a light signal accompanying the detonation wave front[1][5]. This method is point to point to type wherein the first cable signals the start whereas the second cable placed at a known fixed distance stops the timing clock. The fixed distance between probes divided by the timed clock directly gives the VOD value.

\section{Proposed Design Of Sensor}

Worldwide most of the instrument uses continuous wire method for VOD measurement. However this method has limitations of low voltage in resistance per meter which puts limitation that the change in resistance has to be more than $0.4 \%$, also it is susceptible to noise, such method require complex software routine to determine the velocity of detonation. Hence it is proposed to have a sensor wherein the change in resistance per meter is much higher than continuous method which will overcome these two limitations.

The proposed sensor is discrete resistance continuous wire type, which is formed by a series of same value resistances connected in series and supported by a conducting copper wire for providing return current path. A constant current source maintains steady flow of current through the sensor. The highly charged plasma created due to detonation causes the sensor path to get shorted with return line progressively. The overall resistance of sensor thus decreases continuously at the speed of detonation. Since the current through the sensor is held constant, change in resistance gets reflected as change in voltage which is observed and recorded on picoscope. Knowing the sensor construction and correlating it with recorded voltage waveform, the speed of detonation wave can be calculated. In practice it is not always possible to measure VOD of explosive in hole (bulk explosives) hence usual practice is to pack the bulk explosive in cartridge form and perform VOD measurement on it. Cartridge is a single closed bag or tube of circular cross-section containing explosive material. It is punctured from both side wherein from one side the sensor is inserted while from the other side electric detonator

Set up used for experimentation-

\section{Experimentation}

\author{
Settable current source \\ 100 mtrs twisted pair of wire. \\ Picoscope
}

The picoscope and current source were installed in the control room at the distance of about 100m from the blast site. Twisted pair wires were used to connect the sensor and the current source $\&$ picoscope.

The sensor was constructed by connecting MFR resistances soldered to each other in series while maintaining equal distance between them. This series was supported by a thick enamelled copper wire of $1 \mathrm{~mm}$ diameter which also provided the return path for the sensor. The voltage across the sensor was monitored by the picoscope.Velocity of Detonation (VOD) is calculated by distance to time calculation where distance is nothing but the length of the sensor and time is the difference between duration of starting and ending point of the sensor and the VOD calculated is expressed in $\mathrm{m} / \mathrm{s}$. 


\subsection{Case study 1:}

Emulsion cartridge of $25 \mathrm{~mm}$ diameter of company A was selected for the experimentation. For the initiation of explosive a electric detonator was used which was inserted into the cartridge from one end. The sensor was inserted from the other end in the cartridge. The length of the sensor used for testing was $19 \mathrm{~cm}$ in length and discrete resistor string type. Value of each resistor was $10 \Omega$ and the total number of resistors were 17. The distance between the resistances was $1 \mathrm{~cm}$. The constant current source used for the sensor was set at

$22 \mathrm{~mA}$. During the blast the change in resistance of sensor in the form of voltage drop with respect to time was captured in the picoscope. Fig. 2 shows the graph of voltage-time for this test.

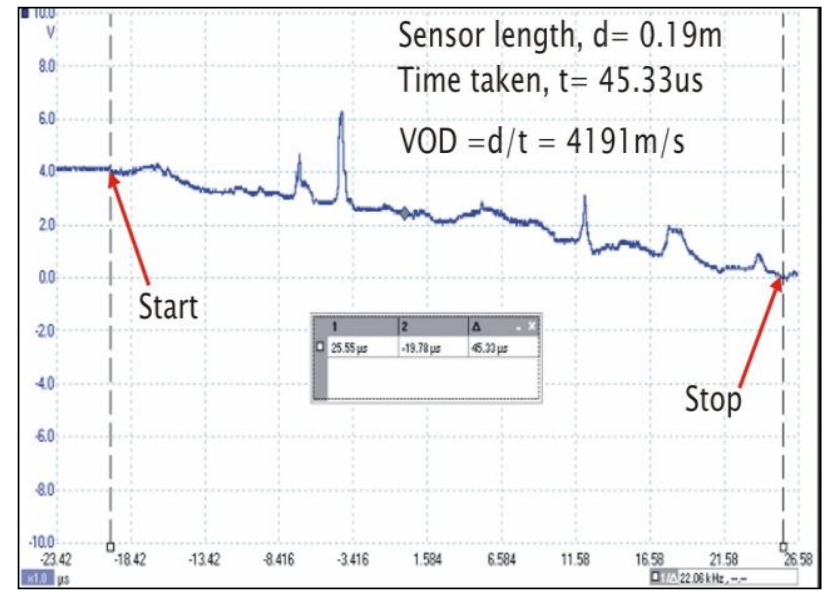

Fig.2 Voltage-Time ( $\mu \mathrm{s})$ graph to calculate VOD

\subsection{Case study 2:}

With the same set-up and explosive of A type the another test were conducted, the simulated graph are shown in Fig.3and 4.

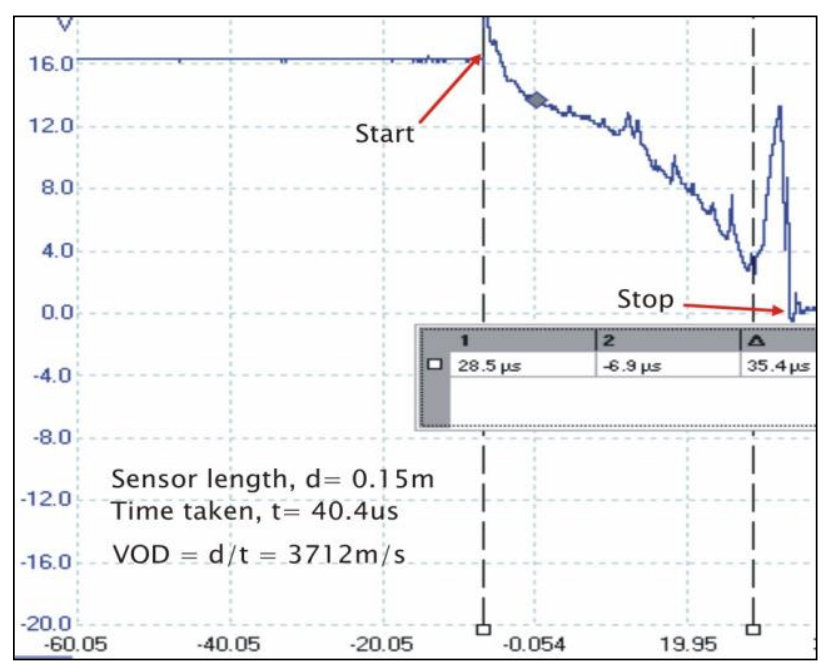

Fig..4 Voltage-Time $(\mu \mathrm{s})$ graph to calculate VOD

It was seen from the test conducted on site and simulation result captured that it is difficult to identify the start and stop point for VOD calculation. Both of these points need to be identified clearly with a few intermediate points. It is observed that there is considerable noise and irregularities in the signal acquired. This is probably due to sensor not being uniform along it's length. The sensor design for this case study is changed that is the value of resistor used for sensor is changed from $10 \Omega$ to $100 \Omega$. The distance between resistors was increased for step change to be seen when the blast occurred.. However this didn't happen and considerable noise observed suggesting further change in the design.

\subsection{Case study 3:}

The initial experiments using continuous wire discrete resistance sensors generated VOD traces that were slightly discontinuous due to the intermittent opening/closing of the contact, making the records difficult to 
interpret as shown in Figure 2, $3 \&$ 4. Proposed design of sensor has small SMD resistors placed at equal distance on a PCB. The sensor was connected to a constant current source. The voltage across this sensor which gets consumed and shorted progressively directly gives VOD $(\mathrm{m} / \mathrm{s})$. Since the resistance per meter or centimetre is known before hand, the rate of voltage drop can directly give VOD.

Test was conducted on the site to calculate VOD with the proposed design of sensor. The sensor length was $10 \mathrm{~cm}$ with resistance value $150 \Omega$ and number of resistances were 10 . Total resistance along the strip of sensor was $1500 \Omega$. Current source of $10 \mathrm{~mA}$ is used to stabilize the initial voltage across the sensor. The initial voltage before starting the blast was about $15 \mathrm{~V}$. Cartridge emulsion explosive of paper tube type of diameter $25 \mathrm{~mm}$ were used for testing. Electrical detonator was placed at one end of cartridge whereas sensor was placed at the other end of the cartridge. Test were conducted with such type of sensor. Test result using this sensor is shown in Fig. 5. It can be seen from these test results that step change is uniform throughout the sensor and noise is reduced to a great extent. Start and stop point is clearly seen in the graph. Effective VOD can be calculated on the basis of start and stop point of sensor consumption.

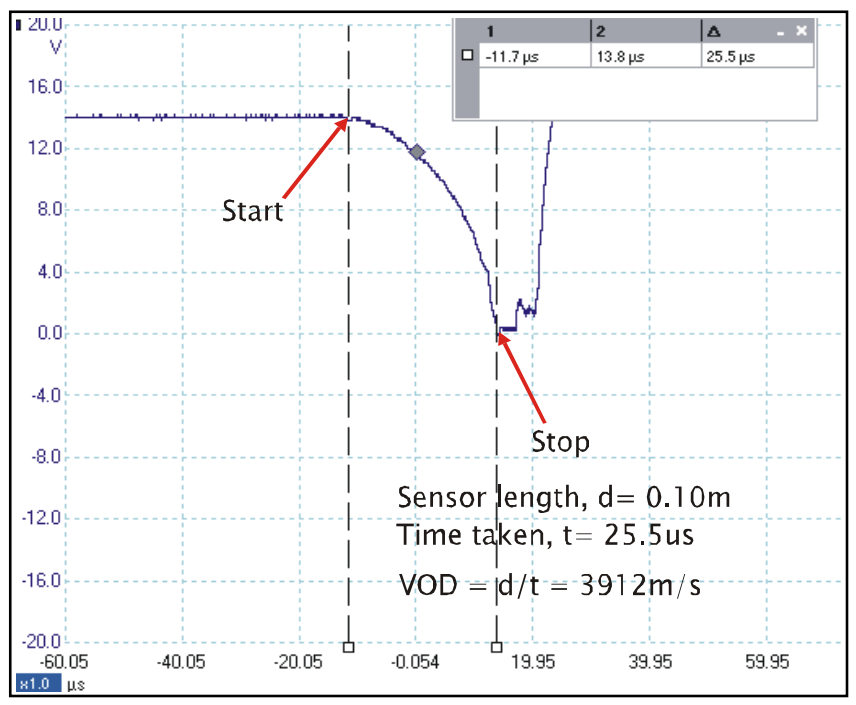

Fig.5 VOD record of SMD sensor in cartridge emulsion explosive $25 \mathrm{~mm}$ paper tube type.

From the test conducted above it was observed that sometimes spikes can be observed wile capturing the velocity of detonation. These were due to inconsistent shorting between signal and return path. To reduce the spikes in the path the sensor design has been changed slightly by making double return path. Total resistance of the sensor was $1100 \Omega$ and the value of each resistor was $100 \Omega$ with 11 number of resistors Length of sensor was $10 \mathrm{~cm}$ and current source of $10 \mathrm{~mA}$ were used to establish the initial constant voltage across the double track return path and strip of resistors. Tests were conducted on cartridge emulsion explosives of $25 \mathrm{~mm}$ diameter. Fig.6 shows the tests results. Start and stop point is easily distinguishable with minimum noise interference.

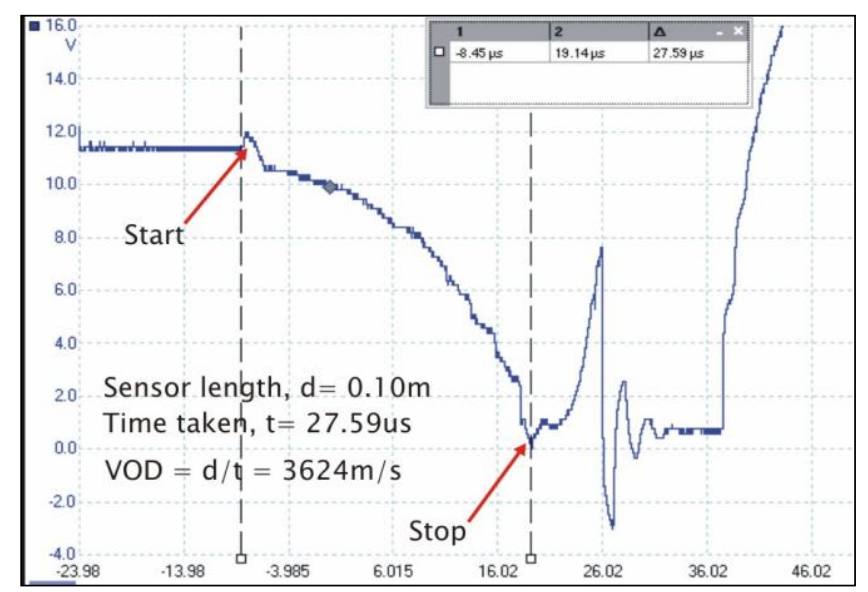

Fig..6 VOD record of SMD sensor in cartridge emulsion explosive $25 \mathrm{~mm}$ paper tube type with double track. 
The various tests conducted on the sites and the observations and results are given in the following Table I.

Table I Design parameter and measured VOD of various case study

\begin{tabular}{|c|c|c|c|c|c|c|}
\hline $\begin{array}{l}\text { Sr.N } \\
\mathbf{0}\end{array}$ & $\begin{array}{l}\text { Fig. } \\
\text { No. }\end{array}$ & Type of Cartridge & $\begin{array}{l}\text { Type of resistors used } \\
\text { for sensor design }\end{array}$ & $\begin{array}{l}\text { Sensor } \\
\text { length(d) }\end{array}$ & $\begin{array}{l}\text { Time between } \\
\text { start \& stop } \\
(\mathbf{t})\end{array}$ & $\begin{array}{l}\text { VOD= d/t } \\
(\mathbf{m} / \mathbf{s})\end{array}$ \\
\hline 1 & 2 & Emulsion $25 \mathrm{~mm}$ & Metal film $1 / 4 \mathrm{~W}$ & $19 \mathrm{~cm}$ & $45.3 \mathrm{us}$ & $4191 \mathrm{~m} / \mathrm{s}$ \\
\hline 2 & 3 & Emulsion $25 \mathrm{~mm}$ & Metal film $1 / 4 \mathrm{~W}$ & $15 \mathrm{~cm}$ & $40.4 \mathrm{us}$ & $3712 \mathrm{~m} / / \mathrm{s}$ \\
\hline 3 & 4 & Emulsion $25 \mathrm{~mm}$ & Metal film $1 / 4 \mathrm{~W}$ & $15 \mathrm{~cm}$ & $40.1 \mathrm{us}$ & $3740 \mathrm{~m} / \mathrm{s}$ \\
\hline 4 & 5 & Emulsion $25 \mathrm{~mm}$ & SMD & $10 \mathrm{~cm}$ & $25.5 \mathrm{us}$ & $3912 \mathrm{~m} / \mathrm{s}$ \\
\hline 5 & 6 & Emulsion $25 \mathrm{~mm}$ & SMD & $10 \mathrm{~cm}$ & $27.5 \mathrm{us}$ & $3624 \mathrm{~m} / \mathrm{s}$ \\
\hline
\end{tabular}

\section{Conclusion}

The various tests conducted do confirm that sensor based on Air Ionization principle give reliable, repeatable and accurate results for in-hole detonation velocity measurement. Also unlike the continuous resistance method, which is presently used world wide, such approach can give required results in a much faster, simpler and ease-to-implement way. In view of the requirement of most of the explosives manufacturer, typical in India, where a simple tool which can directly display VOD and is very easy to use can be a much better proposition than using a continuous VOD system which requires a PC and software analysis to arrive at the VOD values.

These tests conducted also suggest that the sensor shape, size, material used also plays a significant role. It is observed that the PCB based sensor is best suited to measure the velocity of Detonation with reliably, accurately and with much improved consistency. The PCB based sensor also greatly eases the setup procedure.

Another observation is the sensor needs to be protected from moisture in explosives. Further studies and tests need to be done for effective methods for moisture immunity. The sensor and its system needs to be fine tuned for significant step change in voltage level to identify the start and stop conditions.

\section{References}

[1]. Edwin M.Chan, Vivian Lee, Samuel P. Mickan, Phil J. Davies "Low cost optoelectronics devices to measure velocity of detonation", Proceedings of SPIE Vol. 5649(SPIE, Bellingham, WA,2005)

[2]. M. Pradhan, "Effect of Charge Temperature on the detonation Velocity of Bulk Emulsion Explosives" IE(I) Journal-MN vol.90, August 2009.

[3]. "Evaluation of explosive performance through in-the-hole detonation velocity measurement" by National Institute of Rock Mechanics August 2001.

[4]. J.Benterou, E. udd, P.Wilkins, F.Roeske, E.Roos, D.Jackson“'In-Situ Continuous Detonation Velocity Measurements using Fiberoptic Bragg Grating Sensors"

[5]. Lun K. Cheng and Jan van Bree "Fibre-optic sensors in explosion and detonation experiments" SPIE 10.1117/2.1200705.0729

[6]. Jerry.Benterou, Corey V. Bennett, Garrett Cole, D.E. Hare, Chadd May, Eric Udd "Internal Detonation Velocity Measurement Inside High Explosives", SPIE Defence Security and Sensing Orlando, FL, United States April 13, 2009

[7]. M.J.Louw, R.S.Sarracino and S.M.Vather, "Comparison of the theoretical and measured velocities of detonation for selected eexplosives" Journal of The South African Institute of mining and Metallurgy.vol.93, no.6.June 1993 00 147-153.

[8]. Smart structure devices and system II, proceeding of SPIE vol.5649 (SPIE, Belingham, WA2005)

[9]. Gil Smith "ShotTrack VoD system evaluation”, MATRIX Consultants Australia Pty Ltd June 2000. 